\title{
Babesiosis as a Cause of Atraumatic Splenic Injury: Two Case Reports and a Review of Literature
}

\author{
Lily H. Sung, $M D M S^{1,2}{ }^{\infty}$, Ayesha H. Sundaram, $M D^{1,2}$, Andrew L. Glick, MD MPH ${ }^{1,2}$, \\ Daniel F. Chen, $M D^{1,2,3}$, and Linda Shipton, $M D^{1,2,4}$
}

\begin{abstract}
'Department of Medicine, Cambridge Health Alliance, Cambridge, MA, United States; ${ }^{2}$ Harvard Medical School, Boston, MA, USA; ${ }^{3}$ Division of Hospital Medicine, Department of Medicine, Cambridge Health Alliance, Cambridge, MA, USA; ${ }^{4}$ Division of Infectious Diseases, Department of Medicine, Cambridge Health Alliance, Cambridge, MA, USA.
\end{abstract}

\begin{abstract}
We present two cases of Babesia-induced splenic injury at a single institution. In the late summer, two patients presented with left-sided abdominal pain radiating to the shoulder. They were both found to have hemolytic anemia, thrombocytopenia, and acute splenic infarction on imaging. Blood smears showed intracellular ring forms consistent with $\mathrm{Ba}$ besia spp. and low parasitemia $(<1 \%)$. Diagnosis was confirmed by PCR for Babesia microti. Both patients improved with azithromycin and atovaquone, without blood products or surgical intervention. Several weeks following treatment, repeat blood smears revealed no parasites. Splenic infarct and hemorrhage have been previously reported as rare complications of babesiosis. However, given the steady rise in Babesia microti cases in the USA, even these rare complications will become more prevalent. We review both the diagnosis and management of Babesia-induced splenic complications, which can be challenging in patients with low-level parasitemia. Clinicians should consider babesiosis as a cause of atraumatic splenic injury.
\end{abstract}

J Gen Intern Med 36(12):3869-74

DOI: $10.1007 / \mathrm{s} 11606-021-07117-5$

(c) Society of General Internal Medicine 2021

$\mathrm{T}$ wo patients presented to our hospital in Massachusetts within 1 week with splenic injury attributable to babesiosis. With rates of tick-borne illnesses rising, even rare complications will become more prevalent. In this report, we present two cases of Babesia-induced splenic infarct and hemorrhage and review the surrounding literature.

\section{CASE 1}

A 46-year-old healthy man presented to the emergency department in late summer with 2 days of subjective fever and myalgias followed by left-sided abdominal pain radiating to the shoulder. A CT scan of the abdomen and pelvis without contrast was unrevealing. He reported improvement in symptoms with

Received April 12, 2021

Accepted August 25, 2021

Published online September 21, 2021 supportive care and was discharged home. However, 3 days later, the patient returned to the emergency department with worsening abdominal pain.

On presentation, he was afebrile with stable vital signs. He denied alcohol, tobacco, or illicit drug use. He was born in El Salvador but has lived in the USA for many years without recent travel. The patient also denied any recent trauma or known tick bites.

Blood work on admission revealed normocytic anemia with hemoglobin of $11.1 \mathrm{~g} / \mathrm{dL}$ and thrombocytopenia with a platelet count of $116 \times 10^{9} / \mathrm{L}$; the leukocyte count was within normal limits. Hepatic function tests and lipase were normal. Subsequently, a CT abdomen and pelvis with IV contrast showed mild splenomegaly (spleen size $13 \mathrm{~cm}$ ) with multiple wedgeshaped hypo-enhancing areas indicative of splenic infarcts and hyperdense fluid within the abdomen and pelvis concerning hemoperitoneum (Fig. 1). He was admitted to the hospital for further management. Two days later, he required transfer to the intensive care unit for worsening anemia. However, he remained hemodynamically stable, and a $\mathrm{CT}$ angiogram did not show further active contrast extravasation. After reaching a nadir of $8.7 \mathrm{~g} / \mathrm{dL}$ on hospital day 3 , his hemoglobin improved without the need for transfusion or surgical intervention.

Given the acute nature of the splenic infarcts and hemoperitoneum without instigating trauma, a broad workup was initiated. Two sets of blood cultures resulted in no growth. A transthoracic echocardiogram was unremarkable for vegetations or intracardiac thrombus. Testing for EBV, CMV, HIV, COVID-19, Bartonella, Brucella, and Leishmania were all unrevealing. A blood smear was performed and revealed rare intracellular ring forms consistent with Babesia species with parasitemia of $0.1 \%$ (Fig. 2). This was later confirmed by a positive polymerase chain reaction (PCR) for Babesia microti and elevated Babesia microti serologies, IgG 1:320 and IgM 1:160. He was prescribed a 10-day course of oral azithromycin and atovaquone. Subsequent testing for Lyme disease and anaplasmosis were negative by ELISA and PCR respectively.

On follow-up 1 week after discharge, his hemoglobin was improving and the parasitemia on peripheral blood smear had decreased to $<0.1 \%$. By 1 month, the parasite smear was negative and hemoglobin had normalized. 


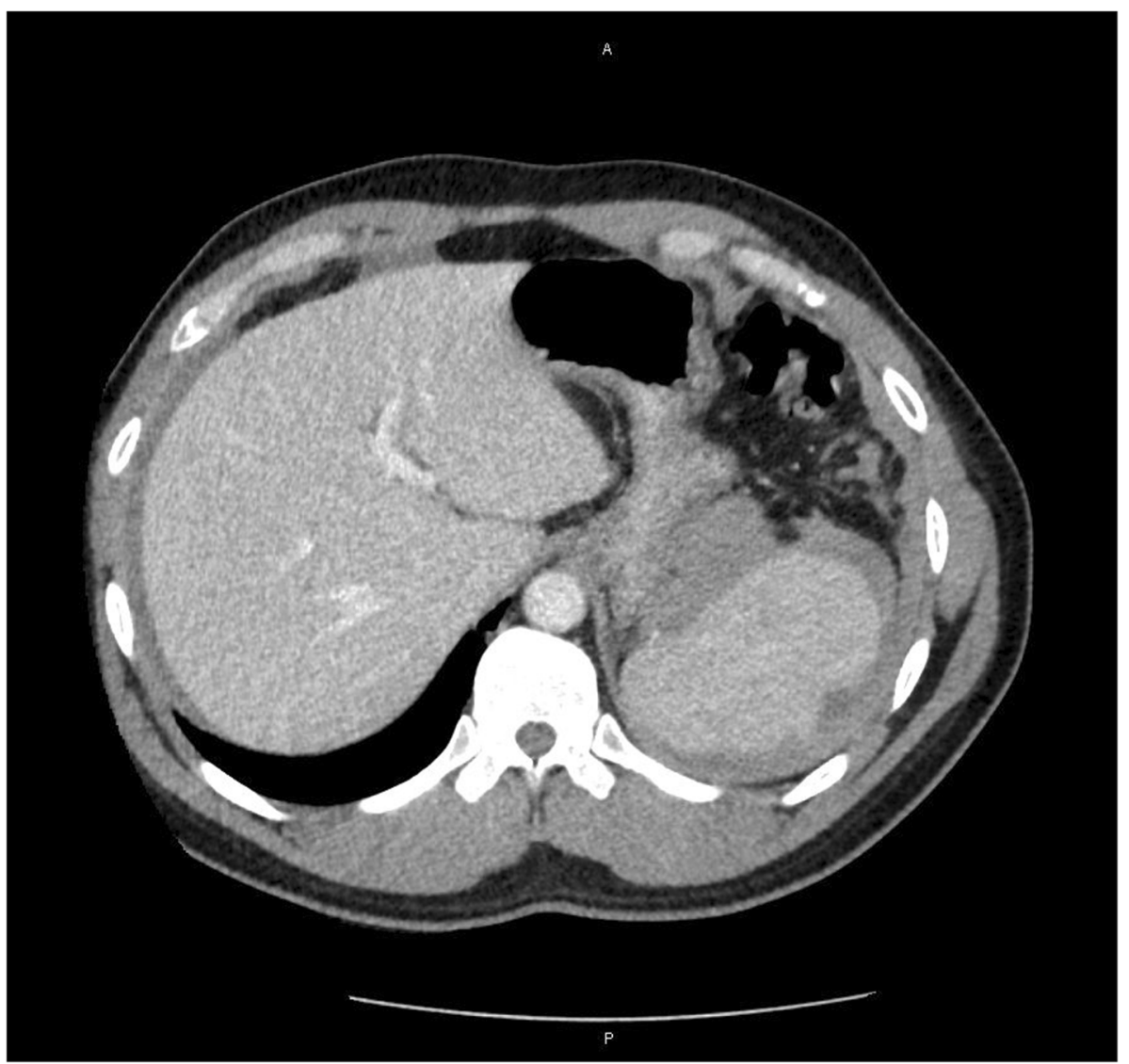

Figure 1 CT abdomen and pelvis with contrast depicting hyperdense fluid enveloping the spleen, indicative of hemorrhage

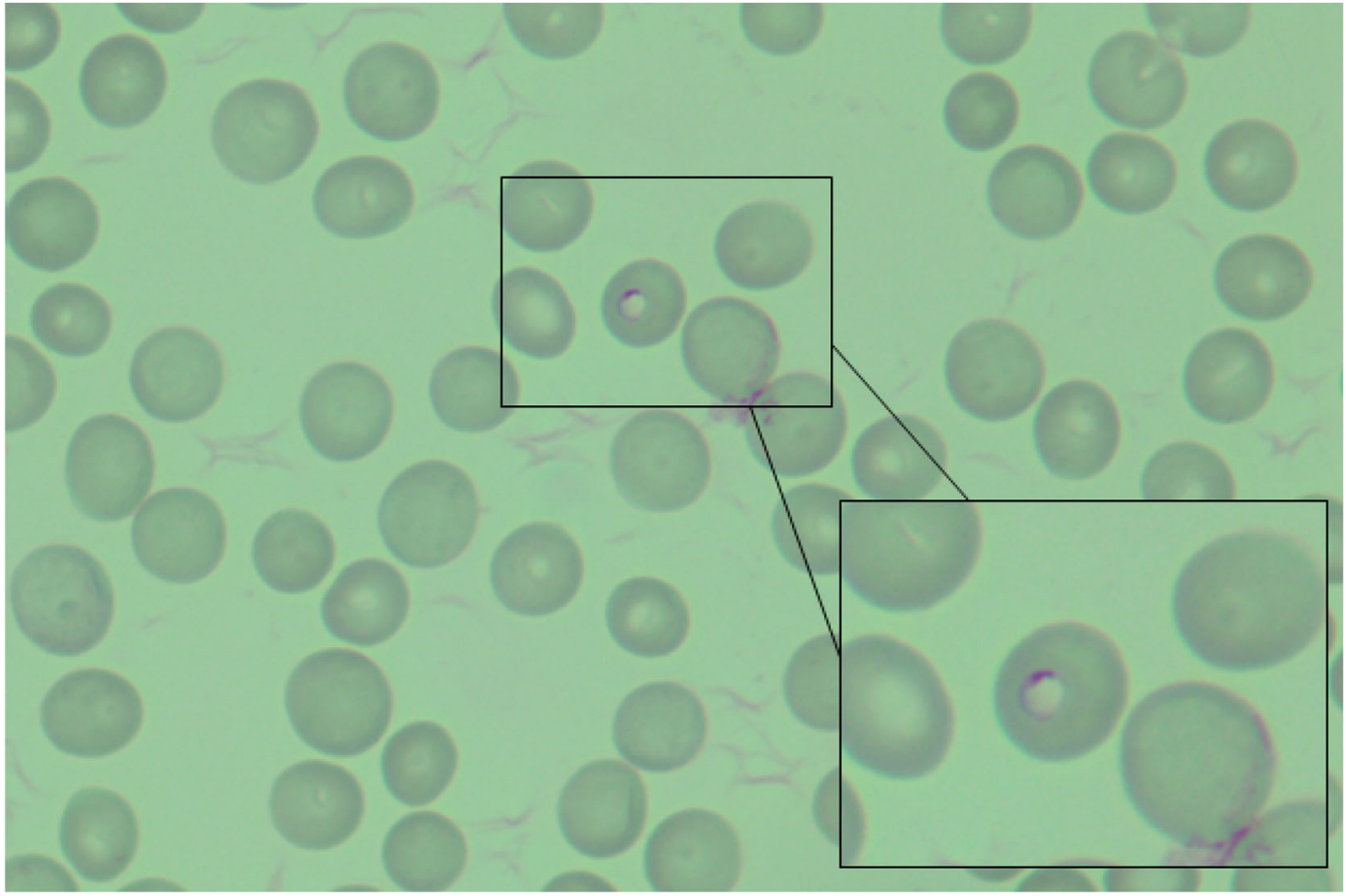

Figure 2 Intracellular ring form in an erythrocyte on peripheral blood smear, consistent with Babesia species 


\section{CASE 2}

A 51-year-old man with obesity and a remote history of gastric bypass surgery presented to the emergency department in late summer with 14 days of fevers, fatigue, and left lower quadrant abdominal pain radiating to the left shoulder. A CT of the abdomen and pelvis with contrast was unrevealing and he was discharged home. However, his symptoms persisted, prompting his return 6 days later.

On re-presentation, he was afebrile with stable vital signs. He took no medications at home and denied alcohol, tobacco, or illicit drug use. He reported traveling to the city of Brasilia, Brazil, several months prior and frequently spending time outdoors in Cape Cod, Massachusetts, with no recollection of mosquito or tick bites. He denied any recent trauma.

Initial blood work revealed normocytic anemia with hemoglobin of $8.7 \mathrm{~g} / \mathrm{dL}$, thrombocytopenia with a platelet count of $100 \times 10^{9} / \mathrm{L}$, total leukocyte count of $7.7 \times 10^{9} / \mathrm{L}$ with $50 \%$ polymorphonuclear cells, and a bandemia of $18 \%$. His hepatic function tests were notable for elevated aminotransferases with AST $336 \mathrm{U} / \mathrm{L}$, ALT $223 \mathrm{U} / \mathrm{L}$, alkaline phosphatase 106 $\mathrm{U} / \mathrm{L}$, and total bilirubin $2.5 \mathrm{mg} / \mathrm{dL}$ with an indirect predominance of $1.7 \mathrm{mg} / \mathrm{dL}$. Haptoglobin was sent and ultimately returned at $<10 \mathrm{mg} / \mathrm{dL}$, suggestive of acute hemolytic anemia. A CT of the abdomen and pelvis with IV contrast (Fig. 3) revealed hepatosplenomegaly (spleen size $13.5 \mathrm{~cm}$ ) with a 4$\mathrm{cm}$ wedge-shaped, hypodense lesion in the lateral spleen that was not present on the CT scan 6 days prior, suggestive of acute infarction.
He was admitted to the hospital. Three sets of blood cultures resulted in no growth, and a transthoracic echocardiogram did not show any evidence of endocarditis or intracardiac thrombus. COVID-19 and HIV tests were negative. Although the city of Brasilia is located just outside the malaria-endemic regions of Brazil ${ }^{1}$, a rapid malaria antigen was sent and was negative. Tests for tick-borne illnesses including babesiosis, anaplasmosis, ehrlichiosis, and Lyme disease were sent. A blood parasite smear revealed intracellular and rare extracellular parasitic organisms (Figs. 4 and 5), consistent with $\mathrm{Ba}$ besia species and parasitemia of $0.6 \%$.

He was started on a 10-day course of oral azithromycin and atovaquone with significant improvement in abdominal pain and fevers. Ultimately, the Babesia microti PCR returned positive, further confirming the diagnosis, and PCR tests for Anaplasma and Ehrlichia returned negative. The Lyme ELISA and Western blot IgM returned positive, indicating acute co-infection with Borrelia burgdorferi, for which he was prescribed doxycycline. A repeat blood smear 2 weeks later showed no parasites; his hemoglobin level also normalized.

\section{DISCUSSION}

Babesiosis is caused by intraerythrocytic protozoa. Infection is most commonly transmitted to humans via a bite from an infected Ixodes scapularis tick, which is endemic in the northeastern USA as well as Michigan and Minnesota. Although over 100 species of Babesia have been reported, the majority

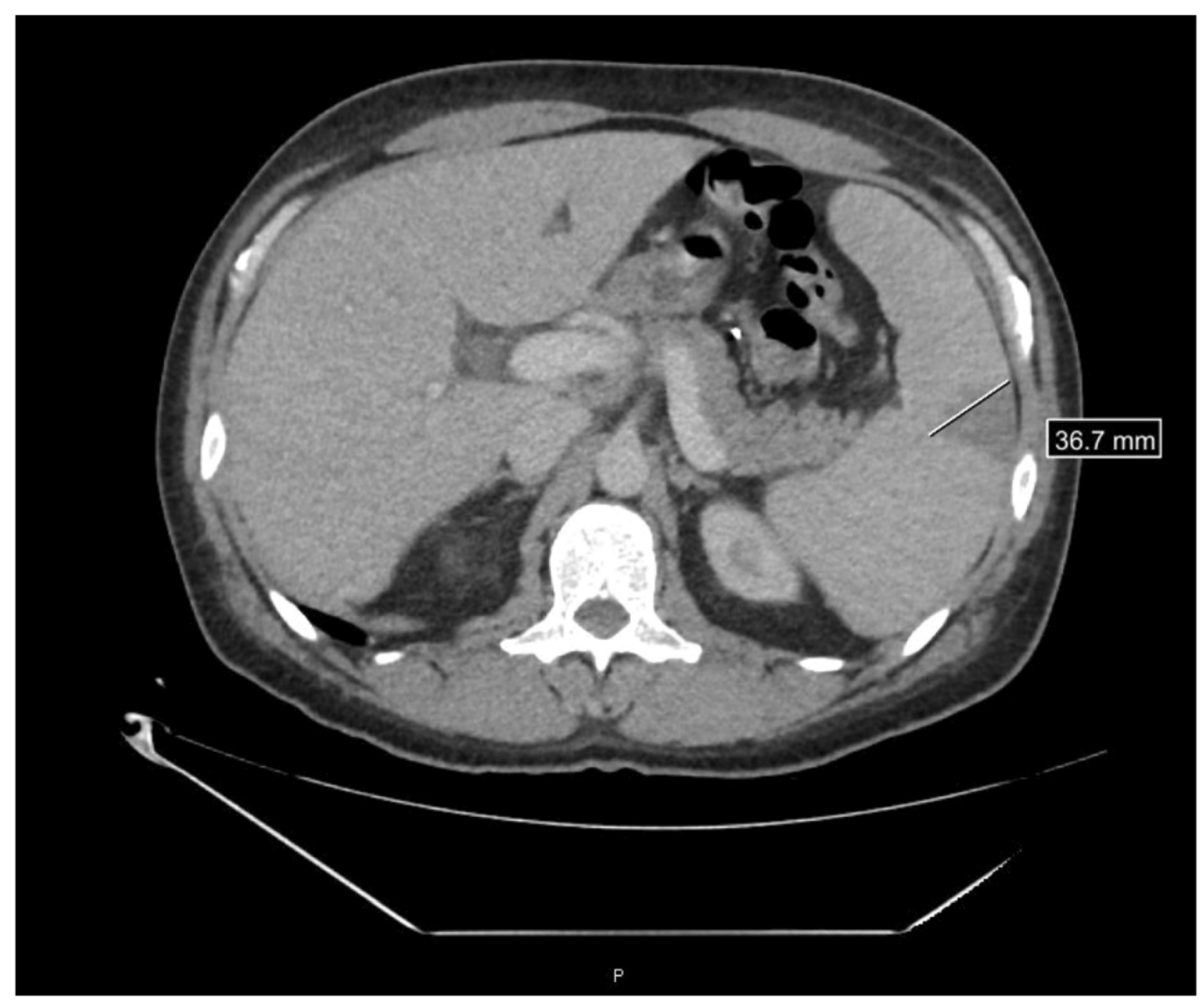

Figure 3 CT abdomen and pelvis with contrast demonstrating a distinctive wedge-shaped, hypodense splenic infarct 


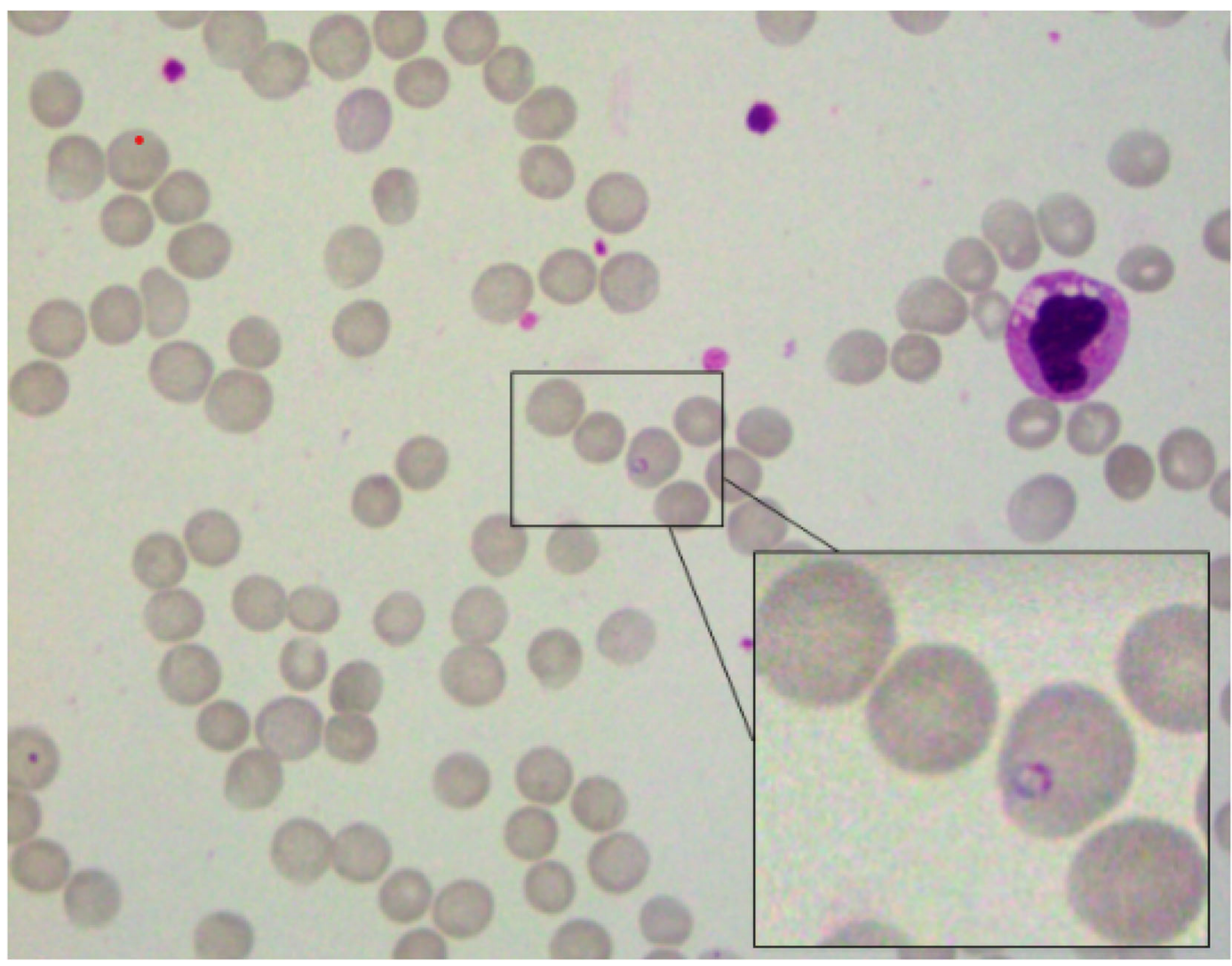

Figure 4 Intracellular ring form in an erythrocyte on peripheral blood smear, consistent with Babesia species

of cases are attributed to B. microti. Currently, 40 states in the USA include babesiosis as a reportable disease and the total number of reported cases has increased steadily. ${ }^{2}$ The rise in cases may be due to increased awareness, testing, reporting, growing deer and tick populations, and increased human-tick interactions in wooded areas. While the highest reported incidence rates are from Rhode Island, Massachusetts, Maine, and Connecticut, sporadic cases are reported throughout the entire USA as people may become infected in an endemic area but are diagnosed after traveling to a non-endemic region. ${ }^{2}$

As highlighted in case 2, patients may be co-infected with more than one tick-borne infection. This may occur through the transmission of multiple pathogens by the same tick or transmission of different pathogens by different ticks in close succession. In addition to the Babesia species, the Ixodes scapularis tick is the vector for other human pathogens including those that cause Lyme disease, anaplasmosis, ehrlichiosis, Borrelia miyamotoi disease, and Powassan virus disease. Because Lyme disease is the most commonly diagnosed tickborne disease in the USA and its geographic distribution overlaps with that of Babesia, it is not surprising that patients with babesiosis may be co-infected with Borrelia burgdorferi. In endemic regions, up to $25 \%$ of patients with babesiosis experience concurrent Lyme disease. ${ }^{3}$

Within the human host, Babesia spp. invade red blood cells (RBCs) and establish an erythrocytic cycle. ${ }^{4}$ Symptomatology of Babesia infection can range from completely asymptomatic to life-threatening illness. It is estimated that about $20 \%$ of babesiosis cases in adults are asymptomatic. ${ }^{5}$ In symptomatic patients, the most common complaints are flu-like symptoms. ${ }^{2}$ Patients who are asplenic, elderly, or otherwise immunocompromised are at the highest risk of severe disease, which can include massive hemolytic anemia, disseminated intravascular coagulation, and multi-organ failure. While the overall death rate is relatively low, around $0.5 \%$, the percent of patients diagnosed with babesiosis who require hospitalization is nearly $50 \%$. $^{2}$ Once hospitalized, mortality rates increase significantly and can reach $21 \%$ in immunocompromised patients. ${ }^{6}$

Splenic infarct and hemorrhage have been reported as rare complications of Babesia. ${ }^{7,8}$ To date, there have been 35 cases of Babesia-induced splenic infarct or hemorrhage reported in the literature. ${ }^{9,10}$

The pathophysiology of this phenomenon remains unclear. ${ }^{9}$ Three proposed mechanisms include (1) increased adhesion of infected RBCs to capillary walls leading to mechanical obstruction of splenic circulation, (2) RBC lysis causing tissue necrosis and endothelial damage, and (3) rapid splenic enlargement due to sequestration of Babesia-infected RBCs and platelets. ${ }^{9}$ Interestingly, like our patients, reports of splenic infarct and/or rupture associated with Babesia tend to be in younger, healthier patients with a lower level of parasitemia compared to others with symptomatic babesiosis. ${ }^{8,9}$ This paradox is thought to be related to the intact cellular immune system of young healthy patients whose spleen is capable of 


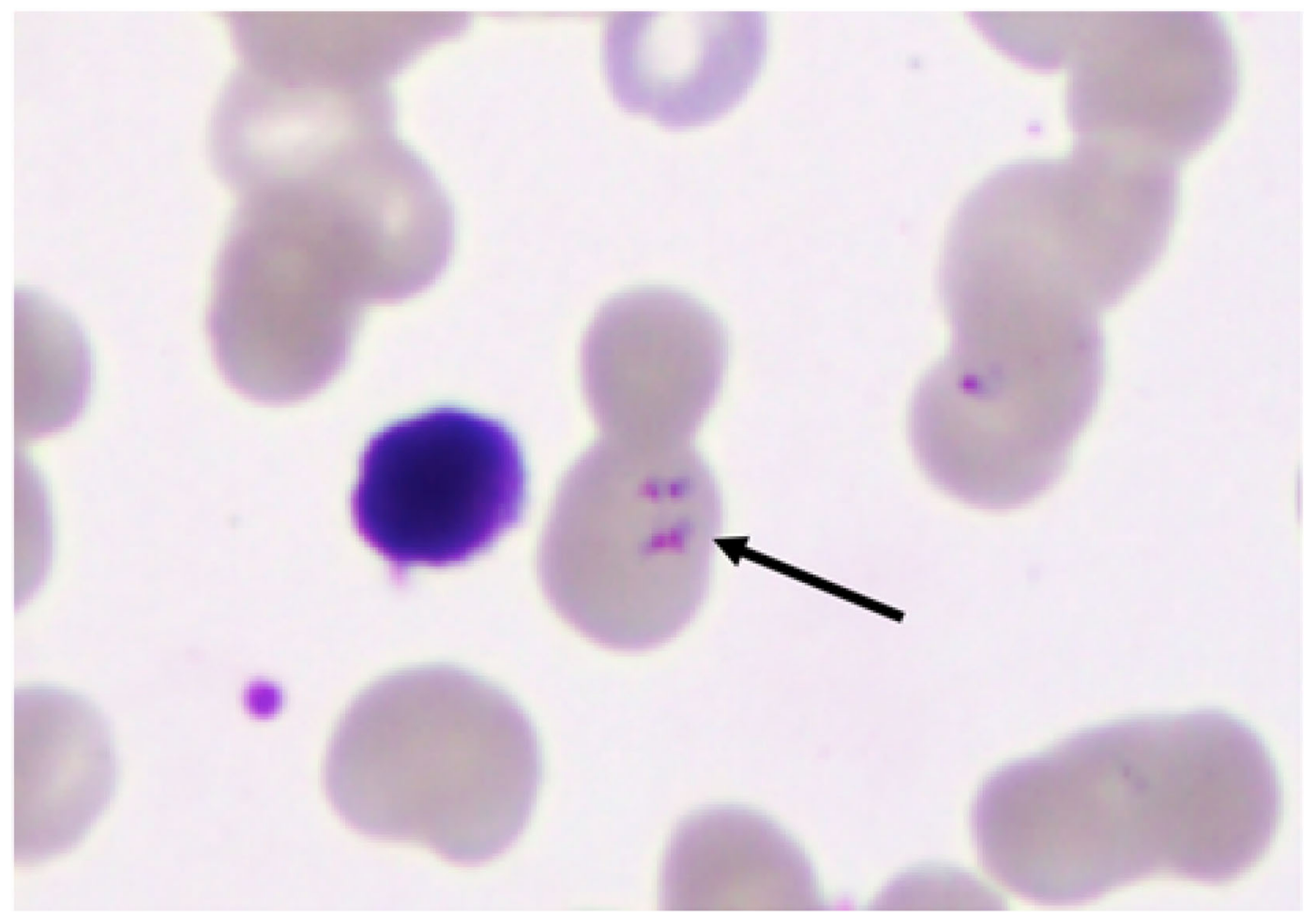

Figure 5 A merozoite tetrad formation known as the "Maltese Cross," which is pathognomonic of Babesia infection

appropriate erythrophagocytosis. This leads to effective clearance of the infected erythrocytes from the bloodstream but may cause increased strain on the spleen. ${ }^{8}$

A presumptive diagnosis of babesiosis can be made in a person with an appropriate exposure history and evidence of hemolysis. Light microscopy to visualize the characteristic ring forms of the intraerythrocytic merozoites on blood smears stained with Wright's or Giemsa stains is the most rapid means of confirming Babesia infection. However, especially in the setting of low parasitemia, which is typical of patients presenting with splenic infarct/hemorrhage, microscopic diagnosis can be difficult. A minimum of 200 to 300 microscopic fields should be reviewed before results are reported as negative and multiple specimens should be examined over several days to increase the diagnostic yield. ${ }^{4}$ Because a larger amount of blood is used in preparing thick films, they can be useful in diagnosing low levels of parasitemia. ${ }^{4}$

The diagnosis can be especially challenging when a patient originates from or has traveled to a malaria-endemic area. Symptomatology including flu-like symptoms, hemolytic anemia, and occasionally splenic infarct or hemorrhage are features shared by both infections. While there is minimal overlap globally between malaria and Babesia-endemic regions, with increased travel, it is not unusual for a patient to present after potential exposure to different areas known to be endemic to malaria and Babesia. On blood smear, Babesia spp. can resemble the early ring from trophozoites of Plasmodium falciparum. Rings of Babesia spp. are identified by delicate cytoplasm and are often pleomorphic, usually vacuolated, and do not produce pigment. ${ }^{2}$ Rarely, the merozoites of Babesia spp. are arranged in a tetrad known at the "Maltese Cross" (Fig. 5), which when seen is considered pathognomonic for Babesia infection. Extracellular parasitic organisms are more common in Babesia infection and visualization of gametocytes or schizonts would confirm malaria infection.

PCR can be used to aid in the diagnosis when blood smears are inconclusive and to differentiate between the various species of Babesia. ${ }^{11}$ However, the turnaround time may vary and PCR is not likely to aid in a rapid diagnosis. Serology is useful but imprecise as symptoms may precede a rise in antibody titer and antibodies may persist after the infection resolves.

The preferred treatment for babesiosis is the combination of azithromycin and atovaquone. ${ }^{12}$ Clindamycin plus quinine is an alternative, but tolerability is poor. Generally, 7-10 days of therapy is sufficient. Longer courses of therapy with follow-up surveillance blood smears are recommended for immunosuppressed individuals as relapse is more common. ${ }^{13}$ Relapse has also been reported after extensive splenic infarction, perhaps resulting from an inability of the damaged spleen to clear the parasite. ${ }^{14}$ Critically ill patients and those with a high level of parasitemia of $>10 \%$ may also require exchange transfusion to rapidly decrease the parasite burden. ${ }^{13}$

About half of Babesia-induced splenic rupture cases reported in the literature were treated conservatively with antimicrobial therapy and supportive care, including transfusion of blood products. ${ }^{9}$ Transcatheter splenic artery embolization has been utilized to control bleeding and to preserve the spleen. Splenectomy should be reserved for those with hemorrhagic shock. ${ }^{11}$ Since patients who present with Babesia 
infection generally reside in or frequent an endemic area, preservation of the spleen is of utmost importance in preventing septic shock and mortality in the event of another Babesia infection. Failure to consider Babesia as the etiology of splenic injury can have severe consequences. If splenectomy is performed but the underlying Babesia is not treated with antimicrobial therapy, the resultant asplenia puts patients at risk of massive hemolysis. ${ }^{11}$

These two cases add to the growing body of literature describing splenic complications of babesiosis. It is important that providers consider Babesia in the differential of those presenting with hemolytic anemia and atraumatic splenic injury.

Acknowledgements: We would like to acknowledge Elizabeth Zulinska, MD, and Julia M Braza, MD, MS, both from the Cambridge Health Alliance Department of Pathology, for the images and review of the blood smears.

Corresponding Author: Lily H. Sung, MD MS; Department of Medicine, Cambridge Health Alliance, Cambridge, MA, United States

\section{REFERENCES}

1. Centers for Disease Control and Prevention. Available at: https:// www.cdc.gov. Accessed March 18, 2021.

2. Centers for Disease Control and Prevention. Parasites - Babesiosis. Available at: https://www.cdc.gov/parasites/babesiosis/. Accessed October 16, 2020.

3. Parveen N, Bhanot P. Babesia microti-Borrelia Burgdorferi Coinfection. Pathogens. 2019;8(3): 117
4. Westblade LF, Simon MS, Mathison BA, Kirkman LA. Babesia microti: from Mice to Ticks to an Increasing Number of Highly Susceptible Humans. J Clin Microbiol. 2017;55(10): 2903-2912.

5. Krause PJ, Mckay $\mathbf{K}$, Gadbaw J, et al. Increasing health burden of human babesiosis in endemic sites. Am. J. Trop. Med. Hyg. 2003; 68(4):431-436.

6. Vannier, E, Krause PJ. Human Babesiosis. NEJM. 2012; 366(25): $2397-$ 2407.

7. Dumic I, Patel J, Hart M, Niendorf E, Martin S, Ramanan P. Splenic Rupture as the First Manifestation of Babesia Microti Infection: Report of a Case and Review of Literature. Am J Case Rep. 2018;19: 335-341.

8. Patel KM, Johnson JE, Reece R, Mermel LA. Babesiosis-associated Splenic Rupture: Case Series from a Hyperendemic Region. Clin Infect Dis. 2019; 69(7): 1212-7.

9. Dumic I, Madrid C, Prada LR, Nordstrom CW, Taweesedt PT, Ramanan P. Splenic Complications of Babesia microti Infection in Junas: A Systematic Review. Can J Infect Dis Med Microbiol. 2020; 2020:6934149.

10. Sahu KK, Petrou N, Cohn Z, Bathini V. Splenic sequela of babesiosis. An International Journal of Medicine, 2020;113(8):577-578.

11. Dumic I, Patel J, Hart M, Niendorf ER, Martin S, Ramanan P. Splenic rupture as the first manifestation of Babesia microti infection: Report of a Case and a Review of Literature. Am J Case Rep. 2018; 19:335-341.

12. Krause PJ, Auwaerter PG, Bannuru RR, et al. Clinical Practice Guidelines by the Infectious Diseases Society of America (IDSA): 2020 Guideline on Diagnosis and Management of Babesiosis. Clin Infect Dis. 2021;72(2): e49-e64.

13. Krause PJ, Gewurz BE, Hill D, et al. Persistent and relapsing babesiosis in immunocompromised patients. Clin Infect Dis. 2008;46(3):370-376.

14. Florescu D, Sordillo PP, Glyptis A, et al. Splenic Infarction in human babesiosis: two cases and discussion. Clin Infect Dis. 2008;46(1):e8-11.

Publisher's Note: Springer Nature remains neutral with regard to jurisdictional claims in published maps and institutional affiliations. 